\title{
Structural transition in bidispersed electrorheological fluids
}

\author{
Xianxiang Huang, Wing Yim Tam, and Ping Sheng* \\ Department of Physics and Institute of Nano Science and Technology, The Hong Kong University of Science and Technology, \\ Clear Water Bay, Kowloon, Hong Kong, China \\ (Received 5 July 2005; published 31 August 2005)
}

\begin{abstract}
We present results from finite element calculations on electrorheological fluids consisting of bidispersed dielectric microspheres and nanoparticles suspended in liquid, with one type much smaller than the other. The face-centered-cubic (fcc) structure of the larger microspheres is shown to be the ground state when the volume concentration of the nanoparticles is between 3-5\%. This is in contrast to the usual situation in which the body-centered-tetragonal (bct) structure is the lowest-energy state. As a function of the nanoparticles' concentration, the bct-fcc structural transition occurs when the nanoparticles' dielectric constant exceeds a threshold value, leading to the electrostatic energy curves of the two structures crossing each other.
\end{abstract}

DOI: 10.1103/PhysRevE.72.020501

PACS number(s): 83.80.Gv, 82.70.-y, 41.20.-q

Electrorheological (ER) fluids are suspensions of dielectric particles in insulating oil. Their rheological properties are controllable by applied electric fields. In theoretical investigations, much attention was paid to systems with uniform-sized microspheres. However, variation in the size distribution, dielectric constant, and physical structure (i.e., coated or noncoated) of dispersed particles can be not only of academic interest, but may also provide a way of varying the ER effect.

For electrorheological fluids consisting of uniform-sized dielectric microspheres, it is known both theoretically and experimentally that under an external electric field, the lowest-energy state of the particles form a body-centeredtetragonal (bct) structure. From theoretical calculations it was also known that a face-centered-cubic (fcc) structure is always a close second, in terms of the electrostatic energy. It is thus an interesting question whether the fcc can ever replace bct as the ground state under some circumstances.

Wu and Conrad [1] have studied experimentally the size effect of particles on ER fluids. By mixing two types of particles, 6 and $100 \mu \mathrm{m}$ diam, they found that the two types of particles do not form chains separately. On the contrary, the chain structure was observed to consist of both types of particles mixed together. The measured yield stress was seen to decrease from the single-sized ER fluid, in agreement with the theoretical prediction of Ota and Miyamoto [2]. Sun and Yu [3] have suggested a dipole-induced dipole model in which there exists the possibility of the bct ground state being unstable. Tan et al. [4] have also pointed out that the field-induced structure might not be chainlike if both the size and dielectric constant effects were taken into account. Instead, particles might prefer to aggregate into clusters or short columns. Wen et al. [5] have studied the bidispersed electrorheological fluid by adding ferroelectric nanoparticles of lead zicronate titanate $(\mathrm{PZT})$ or lead titanate $\left(\mathrm{PbTiO}_{3}\right)$ to ER fluids consisting of $50 \mu \mathrm{m}$ glass microspheres. The static yield stress was enhanced with a peak at certain nanoparticle/

\footnotetext{
*Author to whom correspondence should be addressed. Electronic address: sheng@ust.hk
}

microparticle ratios, for fixed solid/liquid volume fractions.

In this work, we study theoretically the lowest-energy structure for a bidispersed electrorheological fluid consisting of microspheres and nanoparticles, both suspended in a liquid matrix. To include the multipole interaction and the local-field correction, we solve the Poisson equation numerically by the finite element method, and calculate the effective dielectric constant of the composite system under different geometric structural arrangements. It is shown that there can be concentration-induced structural transitions in which the ground-state bct structure of the microspheres is replaced by the fcc structure.

The free energy of the ER fluid in the high-field limit can be expressed as [6]

$$
F=-\frac{1}{8 \pi} \operatorname{Re}\left(\varepsilon_{z z}\right) E^{2} .
$$

Here $E$ denotes the applied electric field, $\varepsilon_{z z}$ is the $z z$ component of the complex effective dielectric constant of the system, and $\operatorname{Re}()$ means taking the real part of the quantity in the parenthesis. Equation (1) translates the problem of determining the ground-state structure into an optimization problem for the dielectric constant. That is, for a given structure formed by the microspheres, what kind of nanoparticle distribution would yield the maximum effective dielectric constant (minimum energy)? Furthermore, what type of microsphere structure can attain the lowest energy, given all possible nanoparticle distributions?

In this work we assumed the nanoparticles to be sufficiently small so that their Brownian motion would preclude the formation of induced structures under the relevant electric-field values. For this to be true, the parameter [7]

$$
\lambda=\frac{\varepsilon_{f}(\beta E)^{2} r_{s}^{3}}{4 k_{B} T}
$$

should be less than 1 , where $r_{s}$ is the radius of nanoparticles, $\varepsilon_{f}$ is the dielectric constant of the fluid, and $T$ is the temperature. Here $\beta$ is the polarizability of nanoparticles, i.e., 


$$
\beta=\frac{\varepsilon_{s}-\varepsilon_{f}}{\varepsilon_{s}+2 \varepsilon_{f}} .
$$

$\varepsilon_{s}$ is the dielectric constant of nanoparticles. This condition imposes an upper bound on the size of the nanoparticles, given by

$$
r_{c}=\left[\frac{4 k_{B} T}{\varepsilon_{f}(\beta E)^{2}}\right]^{1 / 3} .
$$

In our simulation, $\varepsilon_{f}=1, \varepsilon_{s}=20$. For typical values in experiments, we can estimate the maximum electric field to be $E$ $\approx 5 \mathrm{kV} / \mathrm{mm}$, since dielectric breakdown in most materials is likely above this electric field. Hence at room temperature, the upper bound to $r_{c}$ is estimated to be $200 \AA$. We will assume that our nanoparticles are $200 \AA$ or smaller. Compared to the microspheres, the nanoparticles can be treated as infinitesimally small.

When a single nanoparticle is put into the given electric field $\mathbf{E}(\mathbf{x})$ formed by the applied field in the presence of the microspheres, the electrostatic energy change is given by [8]

$$
\Delta W_{e s}=-\frac{1}{2} \int_{V_{1}} \mathbf{P} \cdot \mathbf{E} d V .
$$

Here $\mathbf{P}$ is the polarization density of the nanoparticles and $V_{1}$ is its volume. Thus, it is energetically favorable for the nanoparticles to first occupy the region with the strongest local electric field. Following this line of reasoning, we fill the nanoparticles differentially into the interstitial regions, i.e., nanoparticles are added incrementally, one small portion at a time. Each small $i$ th portion of nanoparticles is filled into the region with the strongest local electric field, evaluated by solving the Laplace equation after the $(i-1)$ th filling. It should be noted that each region has an upper-bound volume concentration of nanoparticles, given by the random tight packing concentration, i.e., 0.63. This differential filling process is continued until nanoparticles are exhausted.

The filling process can also occur outside the interstitial regions of the microspheres. If we assume the region outside of the columns formed by the microspheres to be homogeneous, separated from the columnar region(s) by vertical boundaries, then the electric field in the homogeneous region will always be given by

$$
E=E_{0}=V / L,
$$

if the applied potential difference between the top and bottom plates is $V$, and $L$ denotes the sample thickness along the field direction.

The presence of the nanoparticles in the fluid will locally alter the dielectric constant, calculable from the MaxwellGarnett theory,

$$
\frac{\bar{\varepsilon}_{f}(\mathbf{r})-\varepsilon_{f}}{\bar{\varepsilon}_{f}(\mathbf{r})+2 \varepsilon_{f}}=\theta(\mathbf{r}) \frac{\varepsilon_{s}-\varepsilon_{f}}{\varepsilon_{s}+2 \varepsilon_{f}} .
$$

Here $\bar{\varepsilon}_{f}(\mathbf{r})$ is the local effective dielectric constant and $\theta(\mathbf{r})$ is the local volume fraction of the nanoparticles. When the filling process is finished, the dielectric constant distribution is

$$
\varepsilon(\mathbf{r})=\left\{\begin{array}{ll}
\varepsilon_{l} & \text { if } \mathbf{r} \text { in the large spheres } \\
\bar{\varepsilon}_{f} & \text { if } \mathbf{r} \text { in the fluid }
\end{array} .\right.
$$

Here $\varepsilon_{l}$ denotes the dielectric constant of the microspheres. After the differential filling process is done, the Laplace equation is solved numerically again and the total energy of the column structure is obtained. From this result we can evaluate the effective dielectric constant of the microsphere columnar structure (with the nanoparticles in the interstitial spaces, distributed through the process as described above), given by

$$
\bar{\varepsilon}_{\mathrm{eff}}=\frac{\frac{1}{2} \int \mathbf{D} \cdot \mathbf{E} d V}{\frac{1}{2} E_{0}^{2} V} .
$$

The overall effective dielectric constant of the system is given simply by the following arithmetic average, due to the fact that the columnar structures are parallel to the applied field:

$$
\bar{\varepsilon}_{\mathrm{eff}}=\frac{p_{l}}{p_{\mathrm{str}}} \bar{\varepsilon}_{\mathrm{eff}}^{\mathrm{inf}}+\left(1-\frac{p_{l}}{p_{\mathrm{str}}}\right) \bar{\varepsilon}_{f}^{\text {out }} .
$$

Here $p_{l}$ is the volume fraction of large spheres in the ER fluid and $p_{\text {str }}$ is the volume fraction of large spheres in the column structure, given by

$$
p_{\mathrm{str}}=\left\{\begin{array}{ll}
\frac{2 \pi}{9} & \text { for bct } \\
\frac{\sqrt{2} \pi}{6} & \text { for } \mathrm{fcc}
\end{array} .\right.
$$

$\bar{\varepsilon}_{f}^{\text {out }}$ is the dielectric constant of fluid outside the column structure. It takes the original value of fluid if no nanoparticles are present. Otherwise, it should be evaluated by the Maxwell-Garnett equation (7) according to the nanoparticle concentration.

The calculated effective dielectric constants for bct and fcc structures show a crossover in the lower nanoparticle' concentrations (Fig. 1). It can be seen that the fcc effective dielectric constant exceeds that of bct in the nanoparticle concentration range of $0.03-0.05$, although the difference is small [the enlarged portion is shown in the inset (a) of Fig. 1]. In the following, we give a physical explanation of this crossover.

In the inset (b) of Fig. 1, we plot the effective dielectric constants outside the columnar structures of fcc and bct, respectively. It shows clearly that nanoparticles fill the interstitial regions in the columnar structures first, since in the inset (b) the effective dielectric constants are seen to retain the value of pure fluid $\left(\varepsilon_{f}=1\right)$ before the (nanoparticle) concentration of $0.05 \mathrm{and} /$ or 0.06 is reached. These critical nanoparticle concentrations can be estimated as $0.63 p_{l}\left(1-p_{\text {str }}\right) / p_{\text {str }}$, which gives good agreement with the calculated values to within the discretization error.

It is known that the effective dielectric constant of the fcc columnar structure is larger than that of the bct structure, while the averaged total effective dielectric constant [given 


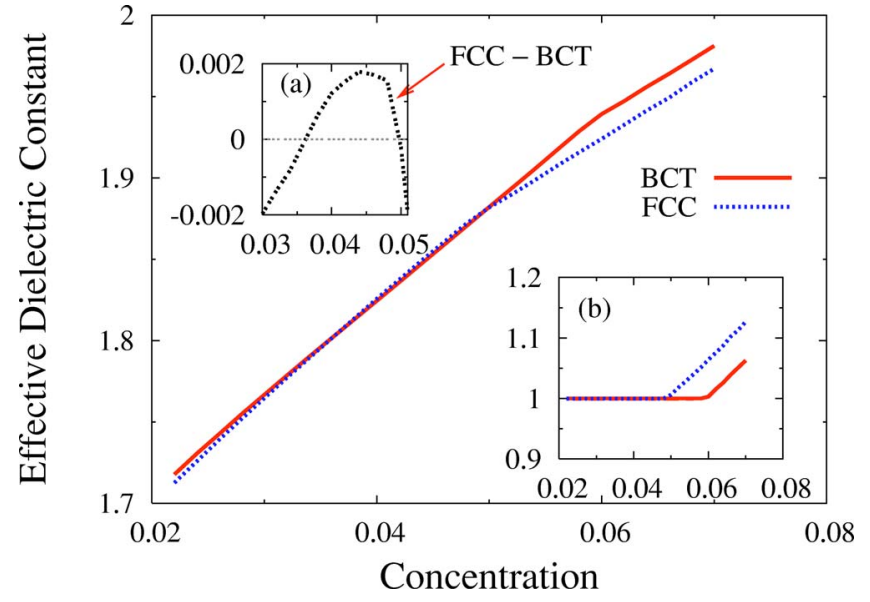

FIG. 1. (Color online) Effective dielectric constants for the fcc and bet structures. In the calculation, the dielectric constant of fluid $\varepsilon_{f}=1$, the dielectric constant of the microspheres $\varepsilon_{l}=4$, and the dielectric constant of nanoparticles $\varepsilon_{s}=20$. The volume fraction of microspheres $p_{l}=0.2$ and the random packing limit is 0.63 . Inset (a) shows an enlarged picture of the crossover region in terms of the difference in the dielectric constant of fcc minus that of bct, and inset (b) shows the effective dielectric constant of the homogeneous fluid region outside of the fcc/bct columnar structures. The kinks indicate the point at which the interstitial space in the columnar structure is full of nanoparticles.

by Eq. (10)] of fcc is smaller than that of bct. This is due to the higher packing efficiency of fcc; hence for the same concentration of microspheres, the volume of the fcc columnar structure is smaller. Therefore, after averaging, the total effective dielectric constant of the bct structure generally surpasses that of the fcc by a very small margin. This is the case studied previously [6]. Now that the overall effective dielectric constant of the fcc structure exceeds that of bct, the columnar effective dielectric constant of the fcc structure must be large enough to overcome its higher packing efficiency. Due to the higher packing density of the fcc structure, the average nanoparticle concentration in the interstitial region is larger than that of bct (for a given overall amount of the nanoparticles), which in turn would cause the average dielectric constant of the nanoparticle-fluid mixture in the interstitial region of the fcc structure, given by the MaxwellGarnett theory [Eq. (7)], to be larger. Hence, the effective dielectric constant enhancement of the fcc columnar structure is much larger than that of the bct structure. If the effective dielectric constant of the fcc columnar structure is large enough so that its overall effective dielectric constant surpasses that of the bct structure, then the fcc columnar structure becomes the ground state. In this regard it should also be noted that the result is also dependent on the ratio $\varepsilon_{s} / \varepsilon_{f}$, which affects the right-hand side of Eq. (7) and reaches a maximum for metallic nanoparticles. If the dielectric constant of the nanoparticles is small, so that the dielectric constant of the mixture in the interstitial region is correspondingly not too large, then the overall effective dielectric constant of the fcc case might not be able to exceed that of the bct case. That is, there is a threshold dielectric constant value for the nanoparticles in order for the structural transi-

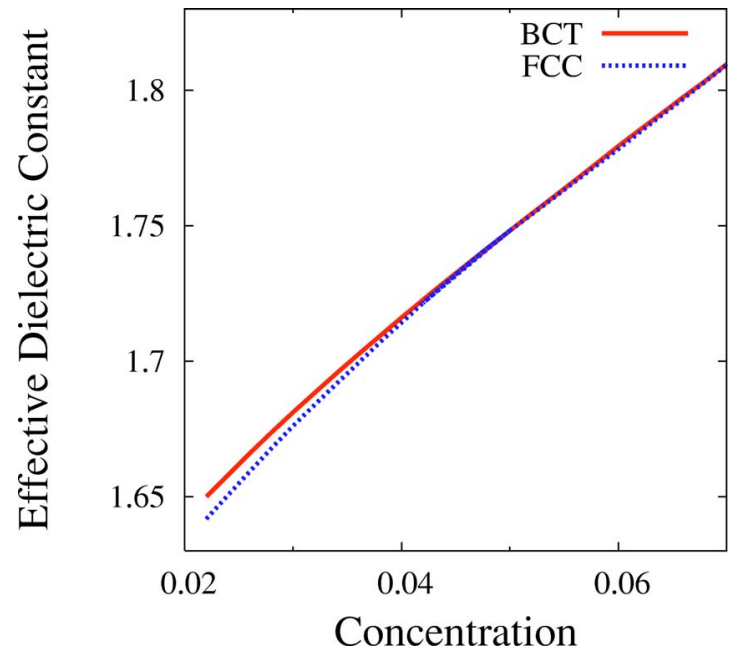

FIG. 2. (Color online) Effective dielectric constants of fcc and bct at $\varepsilon_{s}=6$, close to the threshold value. The two curves are seen to be nearly degenerate.

tion to occur. An estimate of this threshold value is obtained numerically (shown in Fig. 2).

We have also calculated the effective dielectric constant variation when the bct columnar structure is elongated along the electric-field direction, with and/or without nanoparticles being filled within the interstitial regions. The results are shown in Fig. 3. Although the effective dielectric constants increase with the addition of nanoparticles, they decrease more slowly than in the case without nanoparticles, indicating a weaker resistance to stretching, consistent with similar results obtained by [2]. It should be noted here that the present system structure is a balance between the attractive

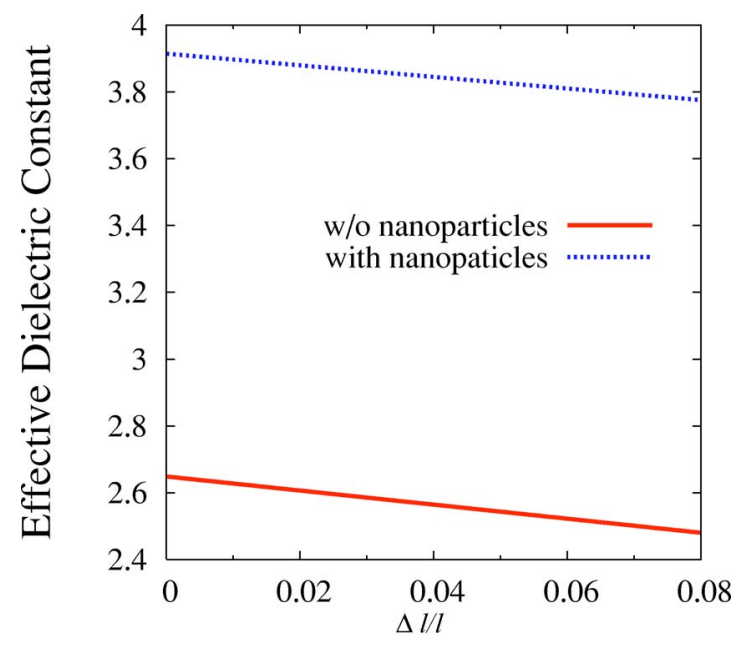

FIG. 3. (Color online) Effective dielectric constant variation of the bct columnar structure when it is elongated (measured by the strain $\Delta l / l)$ along the electric-field direction, with $\varepsilon_{s}=20, \varepsilon_{l}=4$, and the concentration of nanoparticles at 0.048 . The slopes of two curves are -2.11 for the solid curve (without nanoparticles) and -1.74 for the dotted curve (with nanoparticles). It is seen that the required force is larger for the system without the nanoparticles, as evidenced by the larger slope. 
electrostatic force between the microspheres and the hardsphere repulsion when the neighboring spheres touch. Hence if we consider only the electrostatic energy, the variation with strain is linear as seen in Fig. 3, i.e., there is no Young's modulus as such in the present case.

In short, we have found that above a threshold dielectric constant of the nanoparticles in a bidispersed ER fluid, there can be a fcc structure for the ground state within a narrow range of nanoparticle concentrations, between $3-5$ vol \%. It should be noted that if temperature were taken into account, small particles will be distributed in the interstitial spaces with a density given by the Boltzmann distribution. However, if we consider only the high-field limit, then the basic physical picture should remain the same as that described above.

We acknowledge support from RGC Grants No. HKUST6168/01P and HKUST6122/98P.
[1] C. W. Wu and H. Conrad, J. Appl. Phys. 83, 3880 (1998).

[2] M. Ota and T. Miyamoto, J. Appl. Phys. 76, 5528 (1994).

[3] H. Sun and K. W. Yu, Phys. Rev. E 67, 011506 (2003).

[4] Z.-J. Tan, X.-W. Zou, W.-B. Zhang, and Z.-Z. Jin, Phys. Rev. E 59, 3177 (1999).

[5] W. Wen, W. Y. Tam, and P. Sheng, J. Mater. Res. 13, 2783
(1998).

[6] H. Ma, W. Wen, W. Y. Tam, and P. Sheng, Adv. Phys. 52, 343 (2003).

[7] T. C. Halsey, Science 258, 761 (1992).

[8] J. D. Jackson, Classical Electrodynamics, 3rd ed. (Wiley, New York, 1998). 